\title{
Cepstrum Multitaper Method for Optimal Detection of Cognitive Radio Networks
}

\author{
Hebat Allah O. Selim ${ }^{1, *}$, Waheed Sabry ${ }^{2,3}$ \\ ${ }^{1}$ Valley Higher Institute for Engineering and Technology, Science Valley Academy, Egypt \\ ${ }^{2}$ Department of Electrical Power and Energy, Military Technical College, Egypt \\ ${ }^{3}$ Department of Electrical Engineering, Higher Technological Institute, Egypt
}

Received December 9, 2019; Revised January 19, 2020; Accepted February 7, 2020

Copyright $\bigcirc 2020$ by authors, all rights reserved. Authors agree that this article remains permanently open access under the terms of the Creative Commons Attribution License 4.0 International License

\begin{abstract}
Multitaper method (MTM) is an unpopular method that used for spectrum detection. MTM is a good candidate to detect spectrum holes in cognitive radio networks. MTM is accurate, efficient and reliable technique. Cepstrum MTM is described as an effective tool for variance reduction beside its ability in increasing spectrum dynamic range (SDR) due to its isolation property which can be applied to the multiplied mixed signals. As a matter of fact, introducing cepstrum with MTM has achieved better detection performance with the available constraints. In this paper, an algorithm is prepared as a cepstrum MTM for optimal detection and applied to cognitive radio networks. Simple and computationally efficient mathematical expressions of the mean and variance were derived. A closed form expression for the probability of detection (Pd) and false detection (Pf) were obtained accordingly. Analysis of the suggested MTM based cepstrum detection is provided. Different aspects of spectrum sensing parameters were considered in the simulation. A receiver operating characteristic curve is tested where there is a relation between Pd and Pf. The required sample size to achieve certain detection, and the relation of Pd versus signal to noise ratio were also verified. Simulation results were obtained under different affecting factors of the proposed scheme such as probability of false alarm, number of sample points $(\mathrm{N})$, and number of tapers (K). Simulation results reveal that the proposed detector outperforms conventional available tools at the worst scenario operating conditions. The proposed scheme outperforms convention MTM and energy detection by $20 \%$ and $45 \%$ respectively.
\end{abstract}

Keywords Cognitive Radio Networks, Spectrum Detection, Multitaper, Cepstrum, Optimal Detection

\section{Introduction}

Users predict high incoming solutions from $5 \mathrm{G}$ to satisfy the highest demands on the required application. Cognitive Radio [1-4] is a good solution to satisfy those requirements, through finding an efficient under-utilized spectrum to use with the cognitive user. Due to these crucial requirements, spectrum detection [2-5] has been introduced to detect an efficient underutilized spectrum.

Basically, there are many approaches that have been introduced to perform this subtask. They are actually divided into parametric methods and non-parametric methods [2], [6].

Based on this point of view, a parametric method [2], [5] requires a specified statistical model to detect a signal, such as a cyclostationary detector and a matched filter. Furthermore, they need prior information and they are built on the assumption of periodicity of that signal. However, those are deemed as difficult requirements in a cognitive radio. Therefore, the nonparametric method is much applicable to this kind of communication since it does not require a specific statistical model to build a detection model.

In fact, a nonparametric method directly passes the signal to a stochastic model which is capable of giving high resolution without relying on data that belongs to any particular distribution. In other words, it is concerned with the hypothesis of the observed random variables such as wavelet detection, energy detector and multitaper detector. The energy detection (i.e., periodogram) and wavelet detection, they both suffer from a low SDR, which is defined as the ratio between high power spectrum and low power spectrum, where the estimators are concerned with the differentiation between the presence and absence user. Therefore, from the previous non-parametric approaches, MTM method has been chosen as an optimal detector to satisfy detection requirements [2]. 
It uses a set of an orthogonal discrete Prolate Slepian sequence which compromises the bad detection impact resulting from bias variance influence. On the other hand, the Fourier transform of Slepian sequence has a higher energy concentration in the main lobe and a higher reduction in the side lobes. Subsequently, it has a good detection influence. Moreover, Slepian sequence has different variance properties due to its orthogonality property. This means that by averaging all of these sequences, it leads to a low variance rate; consequently, it also has a good detection impact [7], [8].

The following papers [9-14] discussed the set of workable optimization parameters which controls the detection performance. In [10], the author found the applicable range of parameters to achieve the highest probability of detection. In [9], a simple and quick detection algorithm to sense OFDMA signals in a frequency selective fading environment is provided. A closed form of probability of detection, missed detection and false detection are derived to study the impacts of various parameters on the detected performance level. The threshold is not a straightforward equation of probability of false alarm.

In [11], an adaptive threshold for optimal MTM is proposed. Systematic multiple input multiple output MIMO based singular value decomposition is employed. A quadrature form approximation is applied to calculate the estimation equation for MIMO based MTM. The closed form expression of probability of detection and false detection is obtained from Neyman-Pearson hypothesis test. The derived analysis, however, opposes MTM concepts as the mean is not a linear function of tapers and variance does not increase with the number of tapers.

In [12] A cooperative MTM of two-stage spectrum sensing is investigated. For an optimal number of CR users, the analytical expressions are derived to reduce communication overheads. Although it provides enhanced detection accuracy, implementing a cooperative sensing brings numerous drawbacks besides its unreliable implementation in next generation network.

In [13], an optimal detection algorithm for MTM based on Neyman-Pearson theorem and likelihood test ratio is proposed. The proposed detector outperforms and exceeds both conventional energy detection and the cooperative autocorrelation technique. However, it needs large sample size to achieve desired sensing accuracy besides its implementation complexity.

From the previous literature, some models contain unreliable implementation for spectrum sensing in CR network. Other models, on the other hand, provide a reliable implementation and level of performance detection, but the MTM detector faces performance degradation in the worst environmental conditions and specific SNR. Accordingly, our motivation behind this work is to use MTM based cepstrum detection to enhance the reliability and the accuracy of spectrum detection under harsh environmental conditions.

Basically [15], cepstrum comes from the deconvolution problem of two mixed signals or more signals, since it has the ability to extract these components into separate or individual components. Regarding its involved logarithmic functionality, the word cepstrum is derived from the spectrum by converting the first four letters, and its definition is varied according to its functionality [15]-[17]. These definitions will be discussed in section III.

Cepstrum has been employed in numerous areas like in radar and sonar applications. Moreover, it is also applied in mechanical engineering, where it is used to analyze vibration signals from a rotating machine. The other areas of interest are image and speech processing.

In fact, cepstrum is capable of restoring old recording and separating speech into local excitation and system response even if prior data about the system or the source are not given. Actually, this is impossible with traditional methods, which depend on the Fourier analysis of speech signals. Indeed, Fourier analysis converts speech into two multiplying components. This can be a trite solution to isolate the excitation from the system if we know the impulse response of the system. However, in most cases, both components are unknown. Therefore, cepstrum has been investigated to mitigate such dilemma, due to its ability to separate these components without any prior knowledge about any of these.

In addition, cepstrum is investigated in Echo suppression [18], [19]. The echo problem arises from the acoustic reflection that distorts an incident sound. Practically, it is hard to detect and separate an echo from the original signal. Thus, cepstral analysis is applied to detect and eliminate these effects.

The objective of this paper is to further apply and analyze the suggested multitaper based cepstrum detection algorithm. The rest of this paper is organized as follows: Section II describes MTM, section III the cepstrum method is presented. The proposed system model is described and analyzed in section IV. Section V presents the simulation results and discussion conclusions.

\section{MTM}

Considering that we have generated a sample set from the random process $X_{N}$ where $N=\{0,1,2, \ldots . . N-1\}$, those set of samples are multiplied by a number of sequences $h_{K}$ $(\mathrm{N})$. Actually, these sequences are called data tapers (Slepian sequences) where $\mathrm{K}$ is the used tapers, and $\mathrm{N}$ is the sequence length. Furthermore, we take the Fourier transform of this multiplied data sequence. Finally, the $\mathrm{K}$ eign spectrum for the number of taper $\mathrm{K}$ is given by:

$$
Y_{K}(f)=\sum_{t=0}^{N-1} h_{t}^{K}(N, W) X_{t} e^{-2 j \pi f}
$$

We get the estimated power from Thomson theoretical 
[14] equation as follows:

$$
S_{M T M}(f)=\frac{\sum_{K=0}^{K-1} \lambda_{K}(N, W)\left|Y_{K}(f)\right|^{2}}{\sum_{K=0}^{K-1} \lambda_{K}(N, W)}
$$

Equation (2) contains the normalized weighting factor $\lambda_{\mathrm{K}}$ for the selected time to bandwidth product $(\mathrm{N}, \mathrm{W})$, which is related to data taper's power concentration, driven from Eigen solution.

\section{Cepstrum Method}

The cepstrum was defined based on its implementation [19]. The complex cepstrum is defined as the inverse Fourier transform of the logarithmic complex data as in (3).

$$
\begin{aligned}
C_{\text {comp }}(n) & =\frac{1}{2 \pi} \int_{-\pi}^{\pi} \log \left\{X\left(e^{j \omega}\right)\right\} e^{j \omega n} d \omega \\
& =\frac{1}{2 \pi} \int_{-\pi}^{\pi}\left(\log \left|X\left(e^{j \omega}\right)\right|+j \arg \left(X\left(e^{j \omega}\right)\right) e^{j \omega n} d \omega\right)
\end{aligned}
$$

Historically, it has been employed in many applications, since it preserves the signal phase of the composite data. Therefore, it can be applied in waveform recovery, which is known as homomorphic deconvolution or homomorphic filtering. That is used in seismic data, and image processing. It is worth noting that ignoring the phase of spectrum results in the real cepstrum, where it can be written as:

$$
C_{\text {real }}=\frac{1}{2 \pi} \int_{-\pi}^{\pi} \log \left|X\left(e^{j \omega}\right)\right| e^{j \omega n} d \omega
$$

In addition, there are two definitions of power cepstrum, but in the proposed model the definition listed in [16] is applied. It is defined as the absolute of the inverse Fourier transform of the natural logarithm of the power spectrum.

$$
P_{\text {cepstrum }}=\frac{1}{2 \pi} \sum^{N}\left|f^{-1}\left(\log |X(\omega)|^{2}\right)\right|
$$

The most motivating thing about introducing cepstrum with MTM model is its ability to increase SDR due to its additive isolation property which applies to the multiplying mixed signals. This in turn yields to the significant ability in detecting the non-stationary signal with unforeseen harsh environmental conditions, while Fourier transform cannot grantees that especially in harsh environmental conditions.

\section{The Proposed Model}

A non-stationary signal with a discrete sample $\mathrm{X}_{\mathrm{N}}$ where
$\mathrm{N}\{0,1, \ldots . \mathrm{N}-1\}$ is generated from the random statistical process. The signal is multiplied by a number of Slepian sequences $h_{K}$ where $K$ is the number of the used tapers. Then those products are applied to FFT to get a set of Eigen-spectrum sequences, as shown in (1). Moreover, the estimated power is calculated according to (2). The power obtained from cepstrum process is represented as in (6).

$$
P_{\text {cep }}=\frac{1}{N} \sum_{i=0}^{N-1}\left|f^{-1}\left(\log \left\{S_{M T M}\left(f_{i}\right)\right\}\right)\right|
$$

Where $f$ is the Fourier transform, and $f^{1}$ is the inverse Fourier transform. To compare the power obtaining from cepstrum with other systems, the following detection decisions are followed. The detection decision technique can be summarized in two probabilities: the probability of detection $\left(\mathrm{P}_{\mathrm{d}}\right)$ and the probability of false alarm $\left(\mathrm{P}_{\mathrm{f}}\right)$. According to Neyman-Pearson criterion [20], $\left(\mathrm{P}_{\mathrm{d}}\right)$ and $\left(\mathrm{P}_{\mathrm{f}}\right)$ are expressed as:

$$
\begin{gathered}
P_{d}=P\left(\text { Decision } \geq \gamma: H_{1}\right) \\
P_{d}=Q\left(\frac{\gamma-\mu / H_{1}}{\sqrt{\sigma^{2} / H_{1}}}\right) \\
P_{f}=P\left(\text { Decision }>\gamma: H_{0}\right) \\
P_{f}=Q\left(\frac{\gamma-\mu / H_{0}}{\sqrt{\sigma^{2} / H_{0}}}\right)
\end{gathered}
$$

Where $\mu / \mathrm{H}_{1}$ and $\sigma^{2} / \mathrm{H}_{1}$ are the mean and the variance of the received signal power under $\mathrm{H}_{1}$ hypothesis respectively. $\mu / \mathrm{H}_{0}$ and $\sigma^{2} / \mathrm{H}_{0}$ are the mean and the variance of the received signal power under $\mathrm{H}_{0}$ hypothesis, respectively. The power spectrum density of cepstrum power for the received signal represents the chi-square distribution. Therefore, the power distribution function takes the following mathematical model.

$$
f(x ; k)=\frac{x^{\left(\frac{k}{2}-1\right)} e^{\frac{-x}{2}}}{2^{\frac{k}{2}} \Gamma\left(\frac{k}{2}\right)} ; x>0
$$

Where $\Gamma$ is the gamma function that has closed-form values for integer $k$ degrees of freedom, the mean of the estimated power.

$$
\mu / H_{1}=E\left(P_{C e p}\right)=E\left(\frac{1}{N} \log \left(S_{M T M}\left(f_{i}\right)\right)\right)
$$

This equation is approximated to this term, according to [21].

$$
\begin{aligned}
\mu / H_{1} & =\frac{1}{N} \sum_{i=0}^{N-1} \log \left(E\left(S_{\text {MTM }}\left(f_{i}\right)\right)\right)+\frac{1}{N} \sum_{i=0}^{N-1} \log \left(E\left(\chi^{2}\right)\right) \\
= & \frac{1}{N} \sum_{i=0}^{N-1} \log \left(E\left(S_{\text {MTM }}\left(f_{i}\right)\right)\right)+\frac{1}{N} N(\psi(K)-\log (K))
\end{aligned}
$$


Where $\psi(\mathrm{K})$ is the digamma function. The first term can be approximated to according to [13].

$$
\begin{aligned}
& \sum_{i=0}^{N-1} \log \left(E\left(S_{\text {MTM }}\left(f_{i}\right)\right)\right)= \\
& N\left(\log \left(\frac{1}{N}\right) \sum_{i=0}^{N-1} E\left(S_{M T M}\left(f_{i}\right)\right)\right)
\end{aligned}
$$

Therefore, the equation will be:

$\mu / H_{1}=\log \left(\sum_{i=0}^{N-1}\left(\frac{1}{N} E\left(S_{\text {MTM }}\left(f_{i}\right)\right)\right)+(\psi(K)-\log (K))\right)$

Based on [22]:

$$
\sum_{i=0}^{N-1}\left(E\left(S_{M T M}\left(f_{i}\right)\right)\right)=N\left(E_{s}+\sigma_{\omega}^{2}\right)
$$

Where $E_{\mathrm{s}}$ is the estimated power and $\sigma_{\mathrm{w}}{ }^{2}$ is the noise variance. Thus, the final equation will be:

$$
\mu / H_{1}=\log \left(E_{s}+\sigma_{\omega}^{2}\right)+(\psi(K)-\log (K))
$$

Similarly, the mean for null hypothesis will be [22]:

$$
\begin{gathered}
\sum_{i=0}^{N-1}\left(E\left(S_{M T M}\left(f_{i}\right)\right)\right)=N\left(\sigma_{\omega}^{2}\right) \\
\mu / H_{0}=\log \left(\sigma_{\omega}^{2}\right)+(\psi(K)-\log (K))
\end{gathered}
$$

The variance for the two hypothesis process is as follows:

$$
\begin{gathered}
\operatorname{Variance}\left(P_{C e p}\right)=\operatorname{Variance}\left(\frac{1}{N}\left(\log \left(S_{M T M}\left(f_{i}\right)\right)\right)\right)= \\
\frac{1}{N^{2}} \operatorname{Variance}\left(\sum_{i=0}^{N-1} \log \left(S_{M T M}\left(f_{i}\right)\right)\right)
\end{gathered}
$$

The variance for logarithmic of multitaper is derived as in [21].

$$
\text { Variance }\left(\sum_{i=0}^{N-1} \log \left(S_{M T M}\left(f_{i}\right)\right)\right)=N \bar{\psi}(K)
$$

Where $\psi(\mathrm{K})$ is the trigamma function. Thus, the final equation is:

$$
\operatorname{Variance}\left(P_{C e p}\right)=\frac{1}{N} \bar{\psi}(K)
$$

Therefore, the final MTM detection equations:

$$
\begin{gathered}
P_{d}=Q\left(\frac{\gamma-\left(\log \left(E_{s}+\sigma_{\omega}^{2}\right)+(\psi(K)-\log (K))\right)}{\sqrt{\frac{1}{N} \psi(K)}}\right) \\
P_{f}=Q\left(\frac{\gamma-\left(\log \left(\sigma_{\omega}^{2}\right)+(\psi(K)-\log (K))\right)}{\sqrt{\frac{1}{N} \psi(K)}}\right)
\end{gathered}
$$

\section{Simulation Results and Discussion}

In this section, the simulation results for the proposed cepstrum detection are discussed. Under sample size $\mathrm{N}=250$, AWGN channel with $\mathrm{SNR}=-10, \sigma_{\mathrm{w}}{ }^{2}=1$, and testing times $=10000$, the proposed model is validated. The accuracy and verification of the proposed theoretical study was verified by using the following approach. As depicted in fig. (1), the power obtained from cepstrum process is computed according to equation (6) under different simulation conditions. It is worth noting that, the distribution of power per unit frequency is expressed in units of radian /sample, thus the output power is scaled as (pi $x \mathrm{P}_{\mathrm{Cep}}$ ). The simulated mean and variance of the primary users' received (PDF) are obtained from the equation (6) under the Monte Carlo simulation model with at least 10000 times. According to (23), and (24) theoretical threshold $\gamma$, mean and variance are obtained and compared with the simulated ones. Beside this, the proposed model is compared with MTM reported in [22] and with energy detection reported in [23]. 


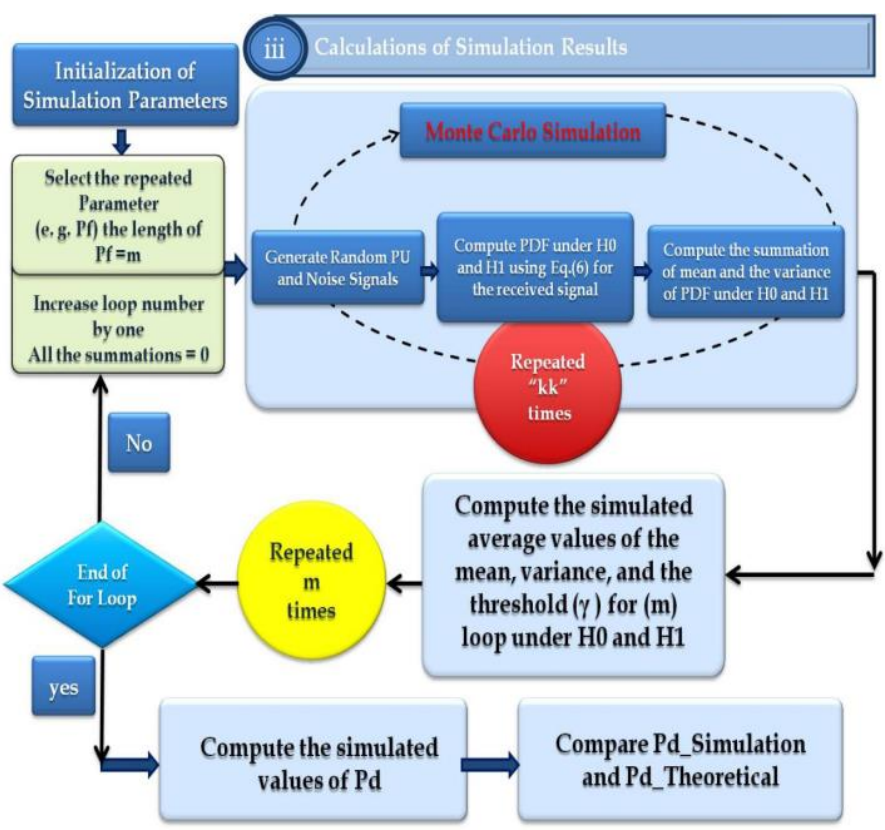

Figure 1. Steps of the verification process

Figure (2) shows the theoretical value of the detection probability along with the simulated one, versus different values of probability of false detection. Under AWGN, and a different number of K, the model is tested. As shown in the figure the detection reaches $90 \%$ at a low value of false alarm $=0.1$. Moreover, the proposed model exceeds the conventional MTM by $20 \%$ at the same condition. At the same occasion, the model outperforms energy detection by $48 \%$. It is clearly shown how the enhancement of SDR impacted on the detection performance. Significantly, these results confirm that the proposed model is an optimal detector for next generation network. Furthermore, the figure also illustrates that the theoretical analysis curve of the detection probability is well-matched with the one generated from the simulation. This also indicates to the implementation reliability of the proposed model. Figure (3) demonstrates how the detection probability behaves under different SNR scenarios. As shown in this figure, the proposed model achieves reasonable detection at very harsh conditions represented at $\mathrm{SNR}=-20$.

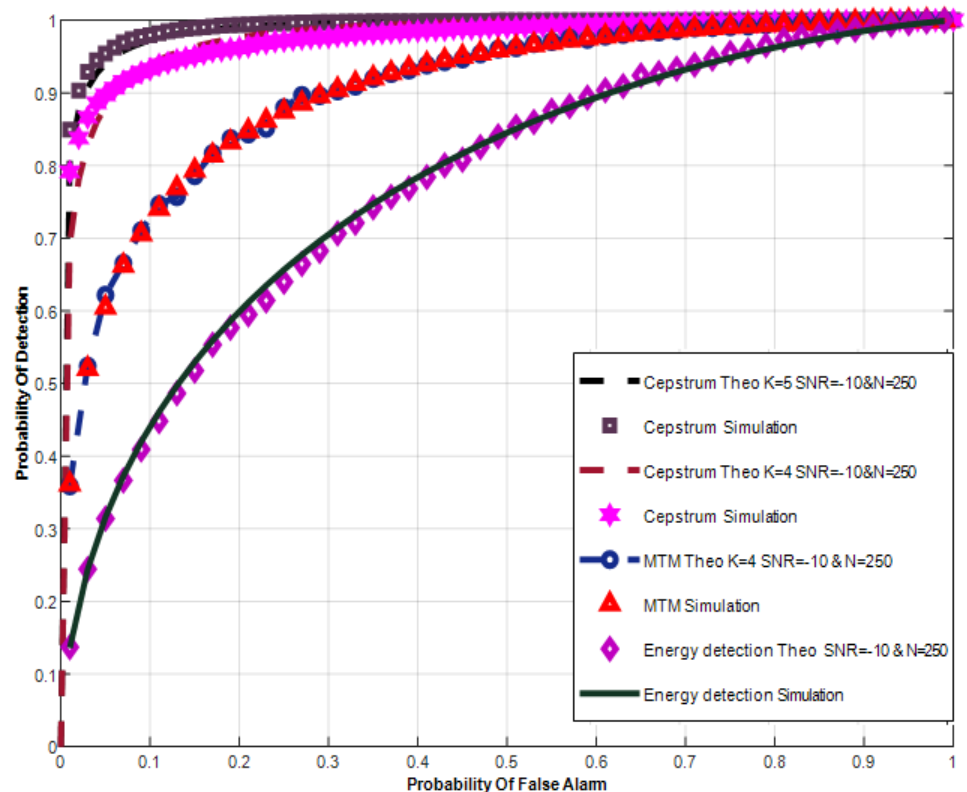

Figure 2. Probability of detection vs probability of false alarm for cepstrum, multitaper and $\mathrm{ED}$ with $\mathrm{N}=250, \mathrm{SNR}=-10 \mathrm{~dB}$ and different values of $\mathrm{K}$

From this case curves start to rise till they reach $90 \%$ at $\mathrm{SNR}=-10$ and this is a good indicator at harsh scenarios like SNR $=-10$. The model also outperforms MTM and energy detection by $18 \%$ and $40 \%$, respectively. These results verify the accuracy of the proposed model under tough environment conditions. 


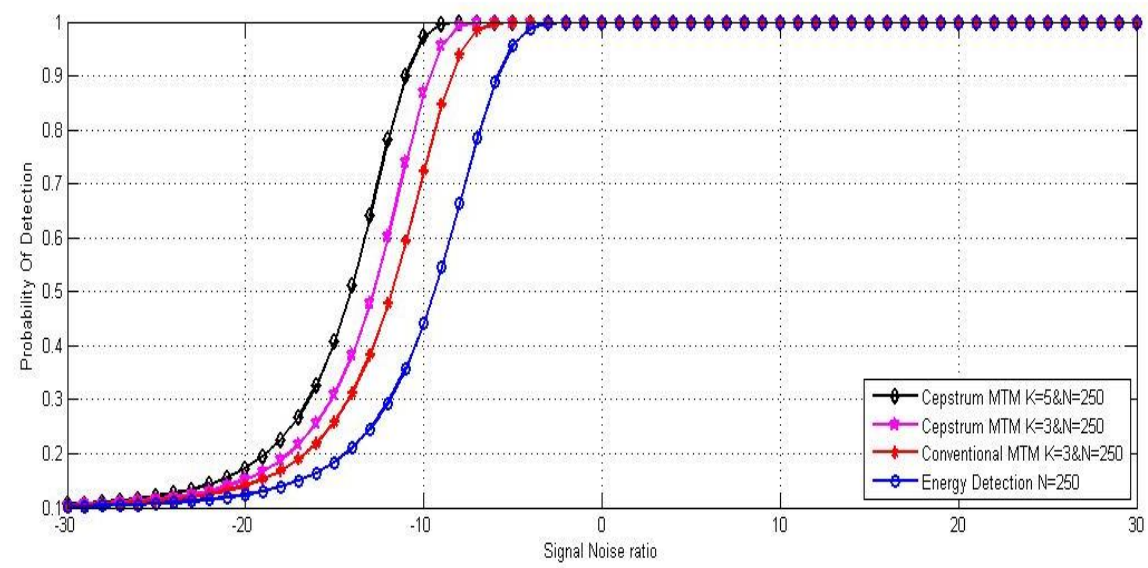

Figure 3. Probability of detection vs $\mathrm{SNR}$ at $\mathrm{Pf}=0.1$ and $\mathrm{N}=250$ for different values of $\mathrm{K}$.

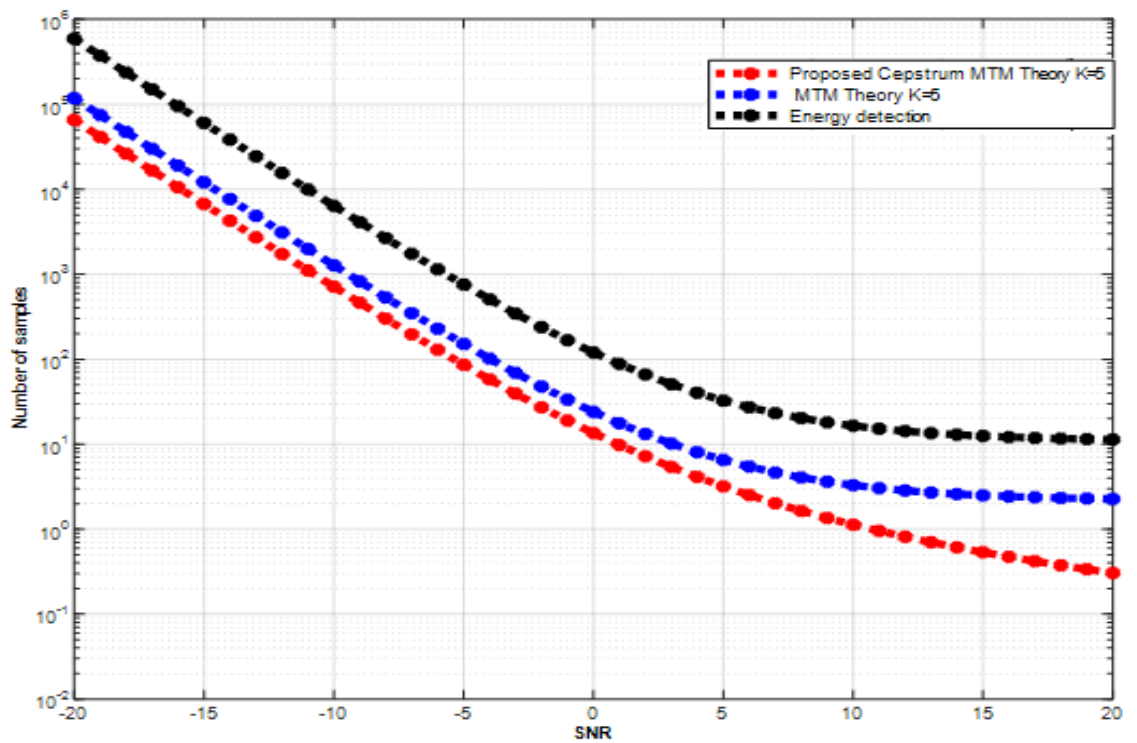

Figure 4. The minimum sample size to achieve the required probability of detection for $\mathrm{K}=5$ as a function of $\mathrm{SNR}$ with $\mathrm{Pd}=0.99$ and $\mathrm{Pf}=0.001$.

Figure (4) represents the minimum required sample size to obtain certain detection probability for cepstrum, multitaper, and Energy detection as a function of SNR under $\mathrm{K}=5, \mathrm{Pd}=0.99$, and $\mathrm{Pf}=0.001$. The figure illustrates that the proposed cepstrum model seeks smaller samples than multitaper and Energy detection in order to achieve the same probability. For instance, with $\mathrm{SNR}=-10 \mathrm{~dB}$, the demanded sample size to achieve detection $=0.99$ is 700 for the proposed cepstrum model, 1300 for multitaper, and 6600 for Energy detection. It is also obvious from the figure that the required samples decrease as the SNR increases. Thus, one sample may be enough to detect the presence of a user; however, in "pmtm" MATLAB function, which is used to estimate multitaper power spectral density, $\mathrm{N}_{\min }$ must be greater than the time-bandwidth product, i.e., $\mathrm{N}_{-}\{\min \}>(\mathrm{K}+1)$. Interestingly, using these small samples size reduces the detection processing time and this is a crucial requirement for CR networks.

\section{Conclusions}

In this paper, multitaper based cepstrum is investigated as a good solution to enhance the detection accuracy at harsh environmental conditions. The mathematical expressions for probability detection and false detection of the proposed detector are derived. Beginning from formulating the Power spectrum density of the proposed model, the mean and variance have been derived, thus the final expression of $\mathrm{P}_{\mathrm{d}}$ and $\mathrm{P}_{\mathrm{f}}$ are obtained. The accuracy of the proposed model has been verified through computer simulation. The results show that the proposed MTM based cepstrum achieves $90 \%$ detection at $\mathrm{P}_{\mathrm{f}}=0.1$. It also shows that the proposed model surpasses MTM and energy detection by $20 \%$ and $45 \%$ respectively. It also depicts how 
the model behaves well under harsh conditions represented at $\mathrm{SNR}=-10$. The simulation results confirm the effectiveness of the proposed method and its reliability as a detector for CR.

\section{Acknowledgements}

The author wants to send the deep grateful to Dr. Mohammed Elgarhy for his advice through the paper.

\section{REFERENCES}

[1] S. Haykin, "Cognitive radio: brain-empowered wireless communications," IEEE journal on selected areas in communications, vol. 23, no. 2, pp. 201-220, 2005.

[2] J. Ma, G. Y. Li, and B. H. Juang, "Signal processing in cognitive radio," Proceedings of the IEEE, vol. 97, no. 5, pp. 805-823, 2009.

[3] S. Haykin, "Fundamental issues in cognitive radio," Cognitive wireless communication networks, pp. 1-43, 2007.

[4] P. Kolodzy and I. Avoidance, "Spectrum policy task force," Federal Commun. Comm., Washington, DC, Rep. ET Docket, vol. 40, no. 4, pp.147-158, 2002.

[5] I. F. Akyildiz, W.-Y. Lee, M. C. Vuran, and S. Mohanty, "Next generation/dynamic spectrum access/cognitive radio wireless networks:A survey," Computer networks, vol. 50, no. 13, pp. 2127-2159, 2006.

[6] P. Stoica and T. Sundin, "On nonparametric spectral estimation," Circuits,Systems and Signal Processing, vol. 18, no. 2, pp. 169-181, 1999.

[7] AO. Salam, RE. Sheriff, SR. Al-Araji, K. Mezher, Nasir Q. Adaptive interacting multiple model-Kalman filter for multitaper spectrum sensing in cognitive radio. Electronics Letters. 2018 Jan 17;54(5):321-2.

[8] M Emara, HS. Ali, SE. Khamis, FE. El-Samie. Spectrum sensing optimization and performance enhancement of cognitive radio networks. Wireless Personal Communications. 2016 Jan 1;86(2):925-41.

[9] Y. Xin, K. Kim, and S. Rangarajan, "Multitaper spectrum detection ofofdma signals in frequency selective fading environment," in Cognitive Radio Oriented Wireless Networks and Communications (CROWNCOM), 2011 Sixth International ICST Conference on. IEEE, 2011, pp. $56-60$.

[10] O. A. Alghamdi, M. A. Abu-Rgheff, and M. Z. Ahmed, "Mtm parameters optimization for $64-\mathrm{fft}$ cognitive radio spectrum detection using monte carlo simulation," in Proc. The Second International Conference on Emerging Network Intelligence (EMERGING 2010), 2010, pp. 107113.

[11] A. O. Salam, R. E Sheriff, S. R. Al-Araji, K. Mezher, Q. Nasir, "Adaptive threshold and optimal frame duration for multi-taper spectrum sensing in cognitive radio," ICT Express. 2019 Mar 1; 5(1):31-6.

[12] B. Ahuja, G. Kaur, "Design of an improved spectrum sensing technique using dynamic double thresholds for cognitive radio networks," Wireless Personal Communications. 2017 Nov 1;97(1):821-44.

[13] T. W. Chiang, J. M. Lin, and H.-P. Ma, "Optimal detector for multitaper spectrum estimator in cognitive radios," in Global Telecommunications Conference, 2009. GLOBECOM 2009. IEEE. IEEE, 2009, pp. 1-6.

[14] D. J. Thomson, "Spectrum estimation and harmonic analysis," Proceedings of the IEEE, vol. 70, no. 9, pp. 1055-1096, 1982.

[15] D. G. Childers, D. P. Skinner, and R. C. Kemerait, "The cepstrum: A guide to processing," Proceedings of the IEEE, vol. 65 , no. 10 , pp. $1428-1443,1977$.

[16] A. V. Oppenheim and R. W. Schafer, "From frequency to quefrency: A history of the cepstrum," IEEE signal processing magazine, vol. 21, no. 5, pp. 95-106, 2004.

[17] J. T. Kim and R. H. Lyon, "Cepstral analysis as a tool for robust processing, deverberation and detection of transients," Mechanical systems and signal processing, vol. 6, no. 1, pp. $1-15,1992$.

[18] R. Martin and C. Burley, "Power cepstrum technique with application to model helicopter acoustic data," 1986.

[19] J. Bolton and E. Gold, "The application of cepstral techniques to the measurement of transfer functions and acoustical reflection coefficients," Journal of Sound and Vibration, vol. 93, no. 2, pp. 217-233, 1984.

[20] S. M. Kay, Detection theory. Prentice-Hall, 1998.

[21] K. S. Riedel, “Adaptive smoothing of the log-spectrum with multiple tapering," IEEE transactions on signal processing, vol. 44, no. 7, pp.1794-1800, 1996.

[22] Heba Allah O. Selim, Heba Y. M. Soliman, Ahmed Ahmed Shaaban Dessouki. "Reliable Analytical Approach for Multi-taper Spectrum Sensing in Cognitive Radio Networks," Port-Said Engineering Research Journal, 2018, 22.2: 56-63.

[23] A. , M. Zulfikar, et al. Enhanced spectrum sensing based on Energy detection in cognitive radio network using adaptive threshold. In: 2017 International Conference on Networking, Systems and Security (NSysS). IEEE, 2017. p. 138-143. 